minutes after the incident. Consciousness is lost after two minutes of anoxia, and irreversible brain damage occurs after four to six minutes. Survival is unusual after immersions of longer than five minutes. Prompt resuscitation is vital; almost all subjects who ultimately survive are making a spontaneous respiratory effort within five minutes after extraction from the water and most do so within two minutes. Children who still require CPR in the emergency department have a poor prognosis: $35-60 \%$ die, and 60-100\% of the survivors are severely brain damaged. Resuscitative measures may achieve somatic survival of the heart and other vital organs but brain function remains severely impaired. The patient in the permanent vegetative state breathes spontaneously and may exhibit random movements but has no purposeful activity or thought. Life expectancy of these children is estimated to average 18 months in institutions and longer in home care. Failure to provide timely resuscitation and lack of knowledge of CPR techniques among pool owners is associated with a poor outcome in drowning and near-drowning incidents. Pentobarbital therapy, at one time thought to be a promising addition to standard therapy for near drowning, has recently been shown to be ineffective. (Wintemute GJ. Childhood drowning and near-drowning in the United States. AJDC June 1990; 144:663-669).

COMMENT. Since the outcame of an immersion event is determined within a few minutes of the onset of the incident, the emphasis is on primary prevention. Mandatory pool fencing and training in cardiopulmonary resuscitation for pool owners should be stressed. Children with epilepsy are often permitted to swim in pools but not in lakes with poor underwater visibility. Strict supervision must be observed since the risks of drowning are several times higher than for the average child.

HYPERGLYCEMIA AND CEREBRAL BLOOD FLOV IN DRONNING

Serial blood glucose levels and cerebral blood flow within 48 hours of admission were correlated with the clinical courses of 20 children with severe near-drowning in the Division of Child Neurology, the Department of Pediatrics, and Section of Neuroradiology, Loma Linda University School of Medicine, Loma Linda, CA. Seven children died, nine were in the persistent vegetative state, and four were normal. Ages ranged from nine month to ten years. Blood glucose levels on admission in the patients who died $(511 \pm 110 \mathrm{mg} \%)$ or who survived in a persistent vegetative state (465 $\pm 104 \mathrm{mg} \%)$ were significantly elevated compared with children who were normal at follow-up $(238 \pm 170 \mathrm{mg} \%)$. The blood glucose values returned to normal by day three of hospitalization. Cerebral blood flow measured by stable xenon computed tomography was significantly decreased in patients who died compared with those who were normal or in a persistent vegetative state. An increase in intracranial pressure was correlated with decreased cerebral blood flow but not with the elevated blood glucose. An elevated initial blood glucose on admission was highly predictive of patients who died or those with vegetative survival. Cerebral blood flow measurements were predictive of eventual 
death but did not differentiate patients who survived in a vegetative state from those who became normal. Cambining blood glucose and cerebral blood flow values improved predictability of outcome in near-drowning. (Ashwal $\mathrm{S}$ et al. Prognostic implications of hyperglycemia and reduced cerebral blood flow in childhood neardrowning. Neurology May 1990; $\underline{40: 820-823) .}$

COMMENT. Cerebral blood flow and blood glucose determinations on admission are useful predictors of the outcome of children with near-drowning and like the Glascow Coma Scale, they are objective measurements of prognosis.

\section{CONGENITAL ANOMALIES}

BRAIN ANOMALIES AND HEART DEFECTS

The type, frequency, and clinical presentation of developmental brain anomalies in 41 infants with the hypoplastic left heart syndrome are reviewed from the Children's Hospital of Philadelophia, and the University of Pennsylvania School of Medicine, Philadelphia. A major or minor central nervous system abnormality occurred in 29\% and included agenesis of the corpus callosum ( 3 cases), holoprosencephaly (1), micrencephaly (9), and cortical mantle malformation (8). Congenital brain anomalies were present in patients either with or without external dysmorphic features that involved the head, face and eyes, fingers, lung and kidney. In patients living only one day or less approximately $50 \%$ had recognizable malformations, microcephaly, and pulmonary anomalies. (Glauser TA et al. Congenital brain anomalies associated with the hypoplastic left heart syndrome.

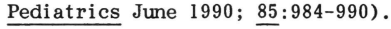

COMNENT. The authors recommend that infants with hypoplastic left heart syndrome deserve careful genetic, ophthalmologic and neurologic evaluations, including CT scan and long-term neurologic follow-up. In the same issue of Pediatrics the authors review their experience with acquired brain lesions associated with hypoplastic left heart syndrome, finding $45 \%$ with hypoxic, ischemic and hemorrhagic lesions secondary to aNS perfusion and glucose oxygen delivery. A duration of cardiopulmonary bypass with hypothermic total circulatory arrest longer than 40 minutes was associated with a higher incidence of acquired neuropathology. (Glauser TA et al. Acquired neuropathologic lesions associated with the hypoplastic left heart syndrome. Pediatrics June $1990 ;$ 85:991-1000).

New management strategies for perinatal hypoxic-ischemic encephalopathy (HIE) are reviewed by Vannucci RC. (Pediatrics June 1990; 85:961-967). Between 10\% and 60\% of asphyxiated neonates with HIE expire during the neonatal period. Of those who survive, $25 \%$ exhibit neuropsychologic deficits. 